\title{
Augmenting the 6-3-5 Method with design information
}

\author{
Andrew Wodehouse \\ University of Strathclyde, James Weir Building, 75 Montrose Street, Glasgow, G1 \\ IXJ, Scotland \\ 0141-548-2091 \\ 0141-548-7986 \\ andrew.wodehouse@strath.ac.uk \\ William Ion \\ University of Strathclyde, James Weir Building, 75 Montrose Street, Glasgow, G1 \\ IXJ, Scotland \\ 0141-548-2091 \\ 0141-548-7986 \\ w.j.ion@strath.ac.uk
}

\begin{abstract}
This paper describes a comparative study between the 6-3-5 Method and the ICR Grid. The ICR Grid is an evolved variant of 6-3-5 intended to better integrate information into the concept generation process. Unlike a conventional 6-3-5 process where participants continually sketch concepts, using the ICR Grid (the name derived from its Inform, Create, Reflect activities and structured, grid-like output) participants are additionally required to undertake information search tasks, use specific information items for concept development, and reflect on the merit of concepts as the session progresses. The results indicate that although the quantity of concepts was lower, the use of information had a positive effect in a number of areas, principally the quality and variety of output. Although grounded in the area of product development, this research is applicable to any organisation undertaking idea generation and problem solving. As well as providing insights on the transference of information to concepts, it holds additional interest for studies on the composition and use of digital libraries.
\end{abstract}

\section{Keywords}

Conceptual design; collaborative design; design methods; digital libraries 


\section{Introduction}

Concept design encompasses the generation of ideas through to the selection of an embodied concept. Associated activities are often undertaken by groups in a collaborative setting and despite the fact this is often an informal process based around sketch work and discussion, a number of formal tools and techniques have been developed to support the process (French 1985, Pugh 1991, Cross 1994, Pahl and Beitz 1995, Ulrich and Eppinger 1995). Although it has been observed that exposure to previous solutions can in some cases lead to fixation on particular approaches (Smith et al. 2008), access to appropriate information, principles, exemplars and context have been shown to be important in creating wellsubstantiated concepts and acting as stimuli for discussion (Benami and Jin 2002, Chuang and Chen 2008). Approaches such as storytelling (Demian and Fruchter 2009), metaphors (Casakin 2007) and inquiry (Eris 2004) have been explored in recent studies as a means to enhance use of information in concept design. Despite investigation of more structured approaches, however, brainstorming and informal collaboration in various forms (Sutton and Hargadon 1996) remains a popular way for designers to rapidly exchange ideas in a non-critical environment and to produce a high volume of conceptual output. This presents a challenge in effectively integrating information to the concept design activity without prescribing a highly systematic approach.

\section{6-3-5 Method: a sketch-based approach}

The 6-3-5 Method (Rohrbach 1969) was developed as an alternative to brainstorming. The name reflects the format, in that a team of 6 participants sketch 3 ideas every 5 minutes. After each five minute round, the concepts are passed round to the adjacent participant. The team is then able to draw on others' ideas for inspiration as they wish. Goldschmidt (1991) has made observations on the importance of sketching in design, emphasising the 'shifts in perception' that occur during this activity with regard to creativity and the development of novel design solutions. While criticisms of conventional brainstorming sessions (Osborn 1953, Kelley 2006) include that they can be personality-driven and lack focus, 6-3-5's use 
of drawing rather than verbal communication (it is also known as 'Brainwriting'), make it an effective alternative. If all participants complete the session properly, a 30 minute session should produce 108 ideas. The results of the session would then be used for further concept development and evaluation. What the method does not do, however, is make use of information to help stimulate and develop ideas. Given its structured and progressive nature, an opportunity was identified to adapt the method to incorporate these elements.

\section{ICR Grid: a sketch + information approach}

As a result, the ICR Grid (the name derived from its Inform, Create, Reflect activities and structured, grid-like output) has been developed. It is a structured method that requires design teams to find and build information resources in parallel with creating solutions. It does, however, maintain the freedom of designers to decide on the direction of exploration by adopting a solution-based approach. Flexible thinking is encouraged by using the different modes of conceptual thinking (analysis, synthesis and evaluation) consistently highlighted in conceptual design literature (Asimow 1962, Cross 1994, Sim and Duffy 2003, Gero and Kannengiesser 2004). The output of the method is a linked grid of concepts and information sources.

In their categorisation of concept design methods, Shah et al. (2000) describe intuitive methods as relying principally on information contained within the team, while systematic methods tend make more use of external information that is applied to the problem. The concept design methods most similar to the ICR Grid are progressive ones such as 6-3-5 (Rohrbach 1969), the Gallery Method (Hellfritz 1978) and C-Sketch (Kulkarni et al. 2001), which provide a similar framework for teams to undertake open-ended design work. The ICR Grid, however, differs in the systematic utilization of information both internal and external to the team (Wodehouse and Ion 2010a). This means it incorporates search activities that other methods would not normally encompass, and furthermore the output is a combination of information and conceptual work, linked and categorized according to the design context. 


\subsubsection{Structure of ICR Grid}

The structure and output of the ICR Grid are shown in Figure 1. It can be viewed as a development of the 6-3-5 Method (Rohrbach 1969), adding a number of new elements to optimize it for more focused concept development. Most importantly, it introduces search tasks in order to help build information context and provide design stimuli. These are rotated around the group and used in the creation of concepts with minimal verbal communication. Another major addition is the competitive element introduced through the use of evaluation - after a concept has been created, it is again passed on to the next participant who reflects on whether the idea is worth developing further. If a positive decision is made, a new information resource is found to apply to the concept and added to the library. If a negative decision is made a new concept is created. This cycle continues for a number of rounds, creating a grid of information and ideas linked by the actions taken during the session.

(a)

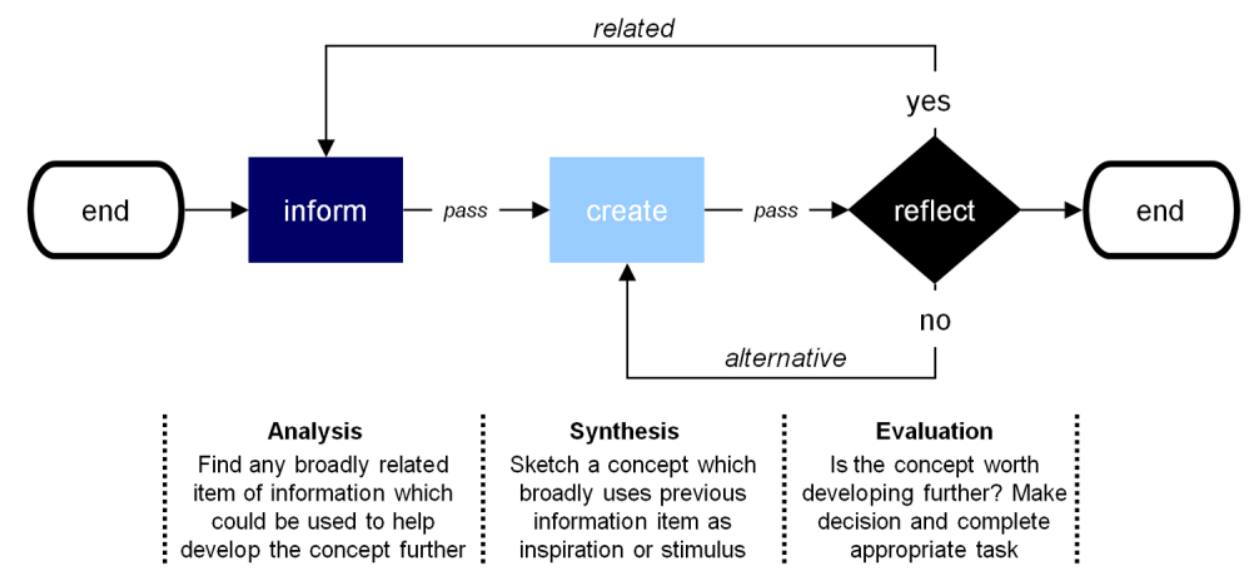

(b)

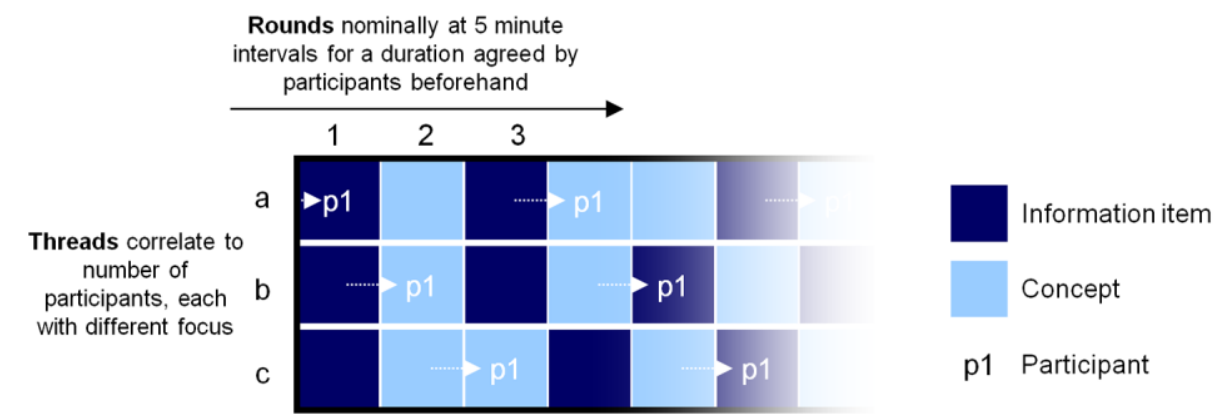

Figure 1: Format of the ICR Grid: (a) flowchart of activity and (b) grid output 


\section{Comparative study}

Since they share many similarities in terms of exchanging sketched concepts in a structured way, it was decided that a comparative study between Rohrbach's 6-3-5 Method would allow the effect of the ICR Grid's enhanced utilisation of information on conceptual output to be analysed. Teams of three were formed randomly from a pool of twenty four senior undergraduate MEng students and postgraduate MSc students, all with an engineering background. In each session, the team had to undertake two 30-minute concept design tasks: one using the 3-X-5 Method and one using the ICR Grid. This provided eight teams, which on reviewing the results was deemed sufficient by the author in providing clear indicators and patterns across sessions. The overall format is shown in Table 1.

\begin{tabular}{ccc}
\hline Session & Using ICR & Using 3-X-5 \\
\hline 1 & & \\
2 & Brief A & Brief B \\
3 & & \\
4 & & \\
5 & & Brief A \\
6 & Brief B & \\
7 & & \\
8 & & \\
\hline
\end{tabular}

Table 1: Format of design sessions

Brief A was to design an ice cream scoop, Brief B was to design a chisel-edge pencil sharpener - simple mechanical devices of similar complexity. The brief for each task specified three key requirements for each design (suitable for one-handed operation, easy to wash etc.) to force participants to consider some design parameters when undertaking the tasks. In order to ensure that the brief was not an unbalancing factor, half the teams used the ICR Grid to tackle Brief A and half used it to tackle Brief B. This allowed discrepancies caused by the brief to be examined.

It was recognised that the dynamics created by personalities would inevitably result in variations in performance across the teams. In addition to having eight sessions to compare, running each session with two tasks allowed internal comparison on how team productivity was affected, i.e. if a team had a high level of 
productivity in relation to the others, there would still be a comparison to be made using the ICR Grid or 3-X-5 Method.

\subsection{Set-up}

The experiments took place in a co-located setting similar to the previous developmental tests, with participants were working face-to-face. Although an important potential application for the structured concept generation approach is the distributed situation, it was felt that a co-located setting provided greater control in terms of experimental set-up and variables, while still allowing adequate evaluation of ICR Grid performance. The set-up, as shown in Figure 2, was almost identical for the two tasks (using 3-X-5 approach, using ICR approach) each team would complete. For the 3-X-5 task, each participant was issued with a briefing document and paper template to complete their concepts. During each round of the session, participants completed sketches in the allocated row of the paper template before passing it to the adjacent participant. The paper templates then continued to rotate around the group in this manner.

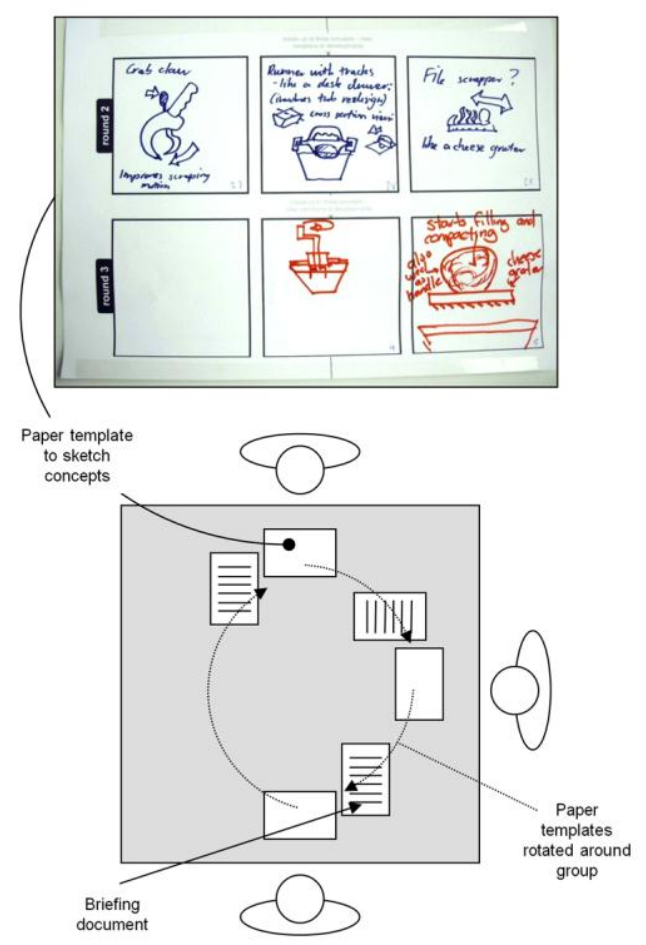

(a)

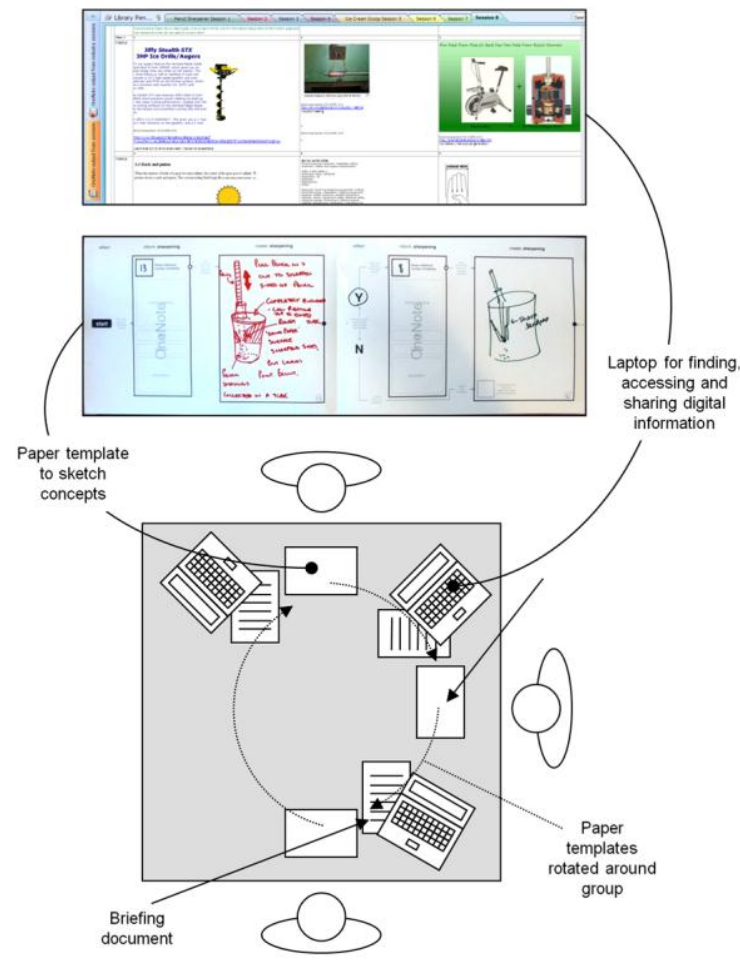

(b)

Figure 2: Experimental set-up for (a) 3-X-5 Sessions and (b) ICR Sessions 
For the ICR Grid task, each participant was issued with a briefing document and similar paper template for completing concepts and circulating around the group, but were additionally issued with a laptop to find and manage digital information. The paper templates were in 'book' form with markers to indicate where digital information had been found. At the end of the session, the books could be opened out and placed in parallel to show overall progression. The software used to manage the shared information resources was Microsoft OneNote, an integrating package that allows users to collect information from a range of sources. The result is an information hub more akin to a designer's notebook than a traditional electronic document, with an informal mix of media. A crucial advantage of OneNote was that it allows a group of people to open and edit a document simultaneously. Utilizing the clipboard feature, which allows areas of web pages to be selected, dragged and dropped into the shared document, was found to be a good way to create a reasonably dynamic and responsive shared digital library. Google was specified as the primary method of searching for information for reasons of familiarity.

\subsection{Experiment variables}

Each team took part in two sessions, one using the 3-X-5 Method and one using the ICR Grid. The aim was to measure the effect of access and use of external information on the teams' conceptual output and keep all the other factors as near to identical as possible.

The metrics used to evaluate the concepts were based on Shah's work on the evaluation of conceptual design performance (Shah et al. 2000, Shah and VargasHernandez 2003). These were identified as providing a robust and thorough review of output, with quantity, novelty, variety and quality recorded. Quantity was easily monitored by totaling the concepts created in each session. Novelty was rated for each idea by comparing the total number of ideas for a particular attribute to the number using a particular principle. Variety on the other hand was applied to the concepts as a group and was measured using a simple genealogy tree for each functional requirement, highlighting different working principles used. Shah's measure of quality was adapted by including the level of sketch detail as a contributing factor in addition to his suggested rating of performance in relation to 
the identified functional criteria. The detail metric was determined by comparing concept sketches for annotation, explanation and sketch complexity with a set of reference concepts adapted from Rogers' (2000) complexity scale. Table 2 summarizes these metrics, and the assessment of each is described in more detail below.

Given the relatively simple nature of the concepts, a panel of experts was deemed unnecessary for evaluation of the concepts. Instead, the ratings assigned were based on the author's own experience and judgement and, having a complete overview of concepts produced during the sessions, every effort was made to be as consistent and objective as possible.

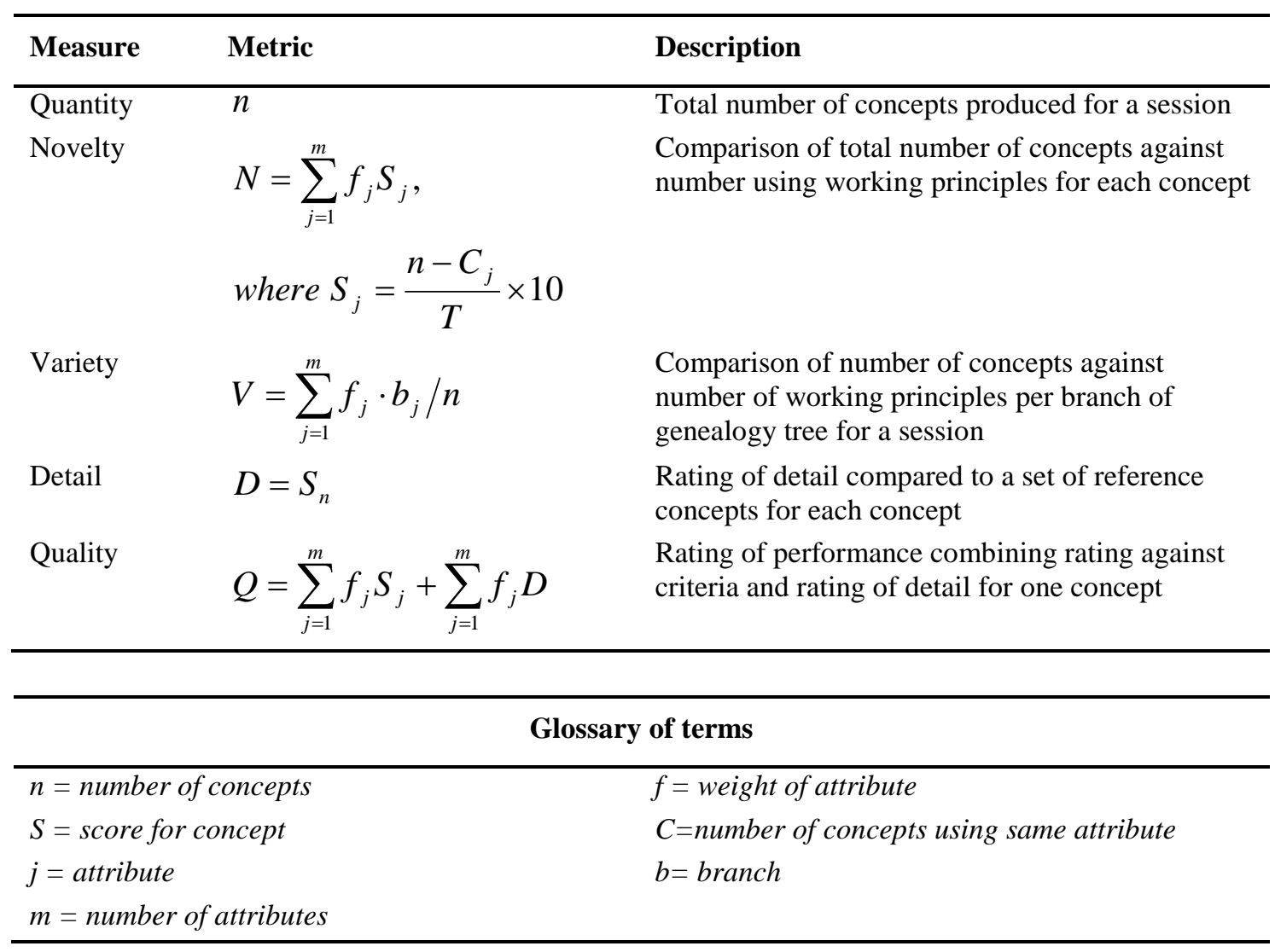

Table 2: Summary of metrics and glossary of terms (after Shah et al.)

\subsection{Session output}

When the results from all eight sessions were compiled, it was found that there was a reasonably strong correlation across them. This is illustrated by the bar graph icons in Figure 3, where the five metrics of quantity, detail, novelty, variety and quality 
were averaged and re-scaled from 0-10 for the concepts produced during the $3-\mathrm{X}-5$ and ICR tasks in each session. It can be noted, however, that Sessions 6 and 7 deviated significantly, with the performance of the ICR Grid in particular being poorer than in the others. The possible reasons for the variation in these sessions are explored below. It was found that the different project briefs had no obvious effect on the concepts produced during the sessions.
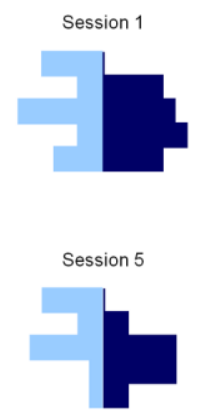
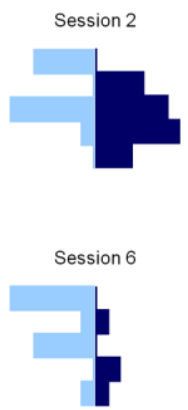

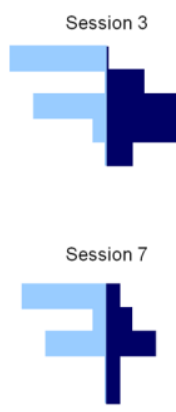

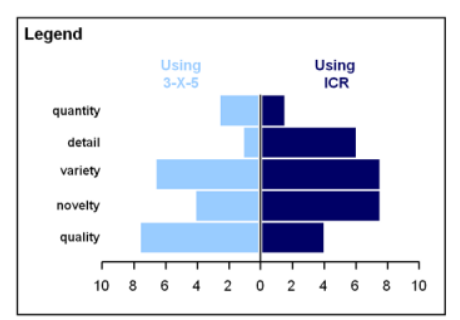

\section{Figure 3: Summary of results}

\subsubsection{Quantity of concepts}

The easiest output of the sessions to monitor, this was simply the total ( $n$ ) number of concepts produced. It was found that the results followed a similar pattern across the sessions, with 3-X-5 producing significantly more concepts than the ICR Grid. This was anticipated prior to the sessions, since the ICR Grid required participants to undertake searching as well as sketching activities, and even then a more methodical approach was required in their construction. This is reflected in the average number of concepts produced in each (38 - 3-X-5, 10 - ICR Grid). When using 3-X-5, participants were asked to sketch as many concepts as they comfortably could in five minutes, rather than demanding three no matter the quality. A properly completed 3X-5 session would have produced 45 concepts. The results are summarised in Figure 4, below. 


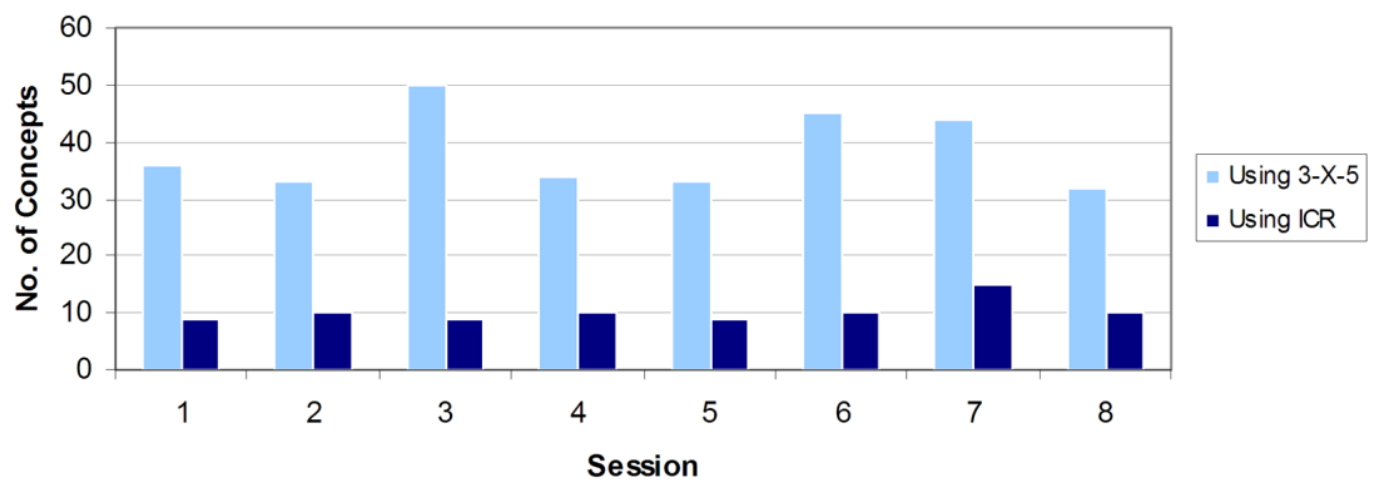

Figure 4: Quantity of concepts produced

\subsubsection{Novelty of concepts}

The measure of novelty was important to show that the ideas produced had a degree of originality. For the two briefs, three attributes were identified as relevant with weightings of $0.4,0.4$ and 0.2 . Each concept was assessed for the approach it had taken to each of the three attributes.

The novelty of each concept was calculated by dividing the number of times the principle was used in the session by the number of concepts produced. The measure of novelty was particularly relevant given that the use of information has been hypothesised as having a positive impact on concept generation. One of the concerns associated with this was that access to previous ideas and concepts may result in ideas which are more derivative, and that encouraging developmental threads may limit scope for blue sky thinking. However, Figure 5 shows that there was a marginal difference in novelty between the concepts produced during the 3-X-5 and the ICR Grid tasks, with the 3-X-5 concepts being slightly higher.

In the ICR Grid, participants created concepts using a comparable number of different attributes, but lacked the occasional 'radical' and often light-hearted idea (for example, a hammer to smash out the ice cream) which emerged during the $3-\mathrm{X}-5$ tasks. This accounts for the marginally higher score for novelty across the $3-\mathrm{X}-5$ tasks. Although these ideas have limited value in that they are unlikely to be developed further, it can be argued that they are important in stimulating creative thinking. It may be that some form of loose idea generation is desirable to encourage 
diverse thinking and act as an information resource prior to the more focussed ICR Grid task. It was particularly apparent when using the ICR Grid that participants continually repeated the same concept, saying 'yes' to its development but making no effort to innovate and improve.

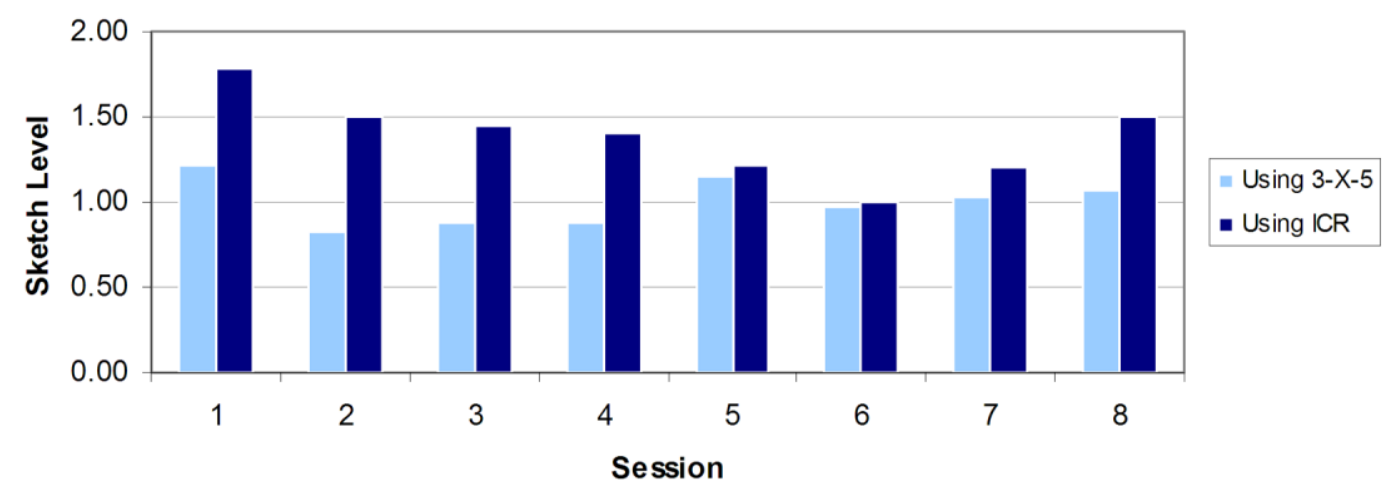

Figure 5: Novelty of concepts produced

\subsubsection{Variety}

Variety differs from novelty in that it applies to a group of ideas rather than the characteristics of an individual idea, and is a measure of the breadth and differentiation between them. Variety was determined using genealogy trees to distinguish the different principles used for the different functional aspects of each concept, with the functions again weighted $(0.4,0.4$ and 0.2$)$ according to importance. The overall measure of variety was then calculated by dividing the number of working principles by the number of concepts for each branch and multiplying it by the weighting function. These were then added to give a total value.

Shah and Vargas-Hernandez (2003) identify four levels of detail for such trees physical principles, working principles, embodiment and detail - but given the limited amount of detail in the concepts produced during the sessions, it was decided to use a simplified genealogy tree consisting of only working principles. It can be seen from Figure 6 that the concepts produced in the ICR Grid showed significantly higher levels of variety than using the $3-X-5$ Method. This can be attributed to the fact that proportionately (although not necessarily as many absolutely) a greater range of principles were applied for the number of concepts produced. Fostering 
separate threads of development to help maintain diversity and introducing new working principles through information stimuli for different working principles meant that for a smaller pool of concepts a greater breadth was addressed. In the 3X-5 sessions, however, it was found that the same principles were often repeated with small variations between them.

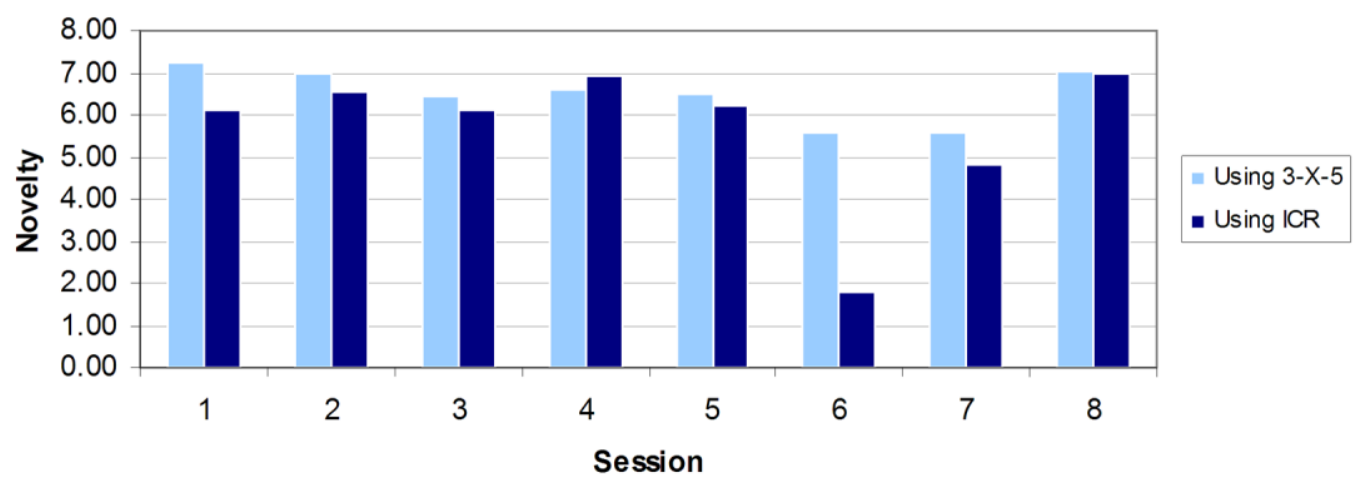

Figure 6: Variety of concepts

\subsubsection{Detail of concepts}

A scale of complexity adapted from Rogers et al. (2000) was used in analysing the level of detail of sketches in each session. The ICR Grid tasks consistently produced concepts that were of a higher level of detail. While the 3-X-5 sessions gave participants to choose how long and how much detail they added to concepts, the access to information, time to complete concepts, and encouragement of clear developmental threads in the ICR sessions were more strongly orientated to concepts with a greater depth of thinking. The results reflect this, and are summarised in Figure 7. 


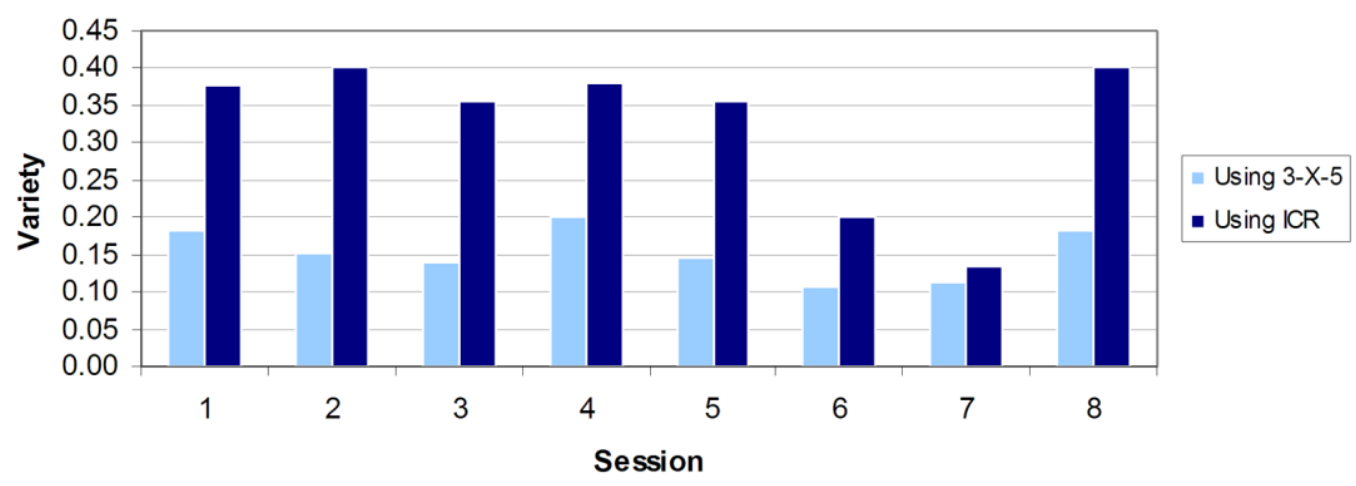

Figure 7: Detail of concepts produced

\subsubsection{Quality of concepts}

A key measure for the sessions was the quality of ideas produced, as the main hypothesis of this research was that better interaction with information would lead to higher quality concepts. As described above, it was decided to make quality a composite of a subjective rating system and the level of concept detail. The functional categories were again weighted and rated individually ( 0 - not addressed, 1 - poor, 2 - okay, 3 - good) according to a combination of the perceived originality and feasibility of the concept embodiments based on the researcher's own design experience. Having a complete overview of concepts produced during the sessions, every effort was made to be as consistent and objective as possible. Detail was introduced as a factor in the calculation of quality as it is a powerful indicator for depth of thinking associated with a concept. The ratings and annotation count were then combined give a score for each concept and averaged to give an overall score for each session. It was found that quality was consistently better in the ICR Grid tasks (Figure 8). This reflects that fact that participants were encouraged to implement information, reflect on validity, and develop promising threads during the task. 


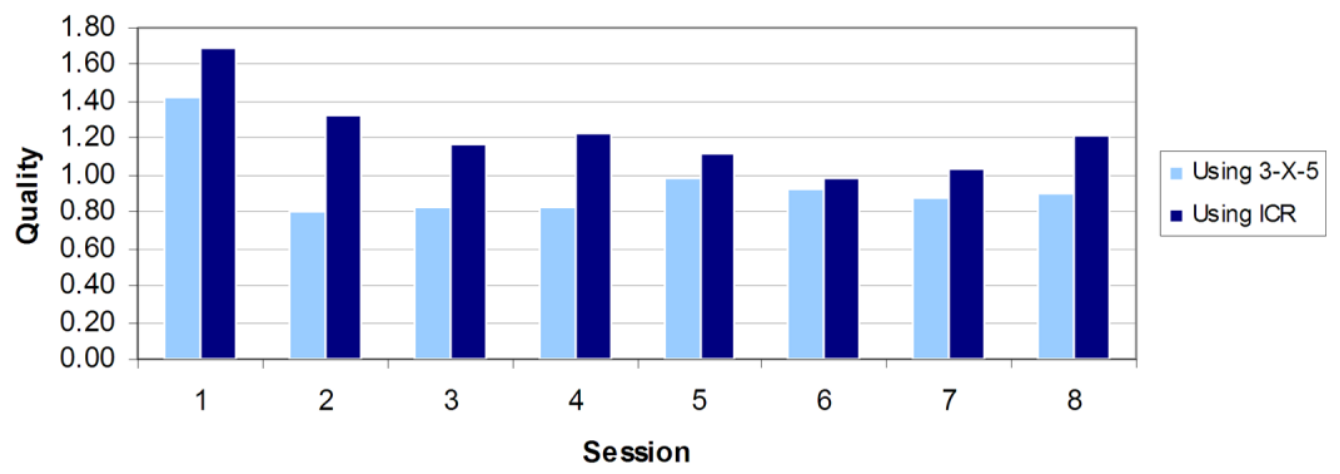

Figure 8: Quality of Concepts

\section{Analysis and discussion}

Although a number of consistent patterns emerged in the results, Session 6 and 7 were identified as deviating noticeably from the other sessions. On exploring the output of these sessions a number of issues have been acknowledged relating to the mechanics of the ICR task to explain why they were particularly poor. These included a propensity to simply say 'yes' to developing concepts without rigorous evaluation, failing to find adequate information sources to motivate and inform new threads of development, and a lack of sketching skills which led to limited communication through annotation.

While these issues were noted as significant for the performance and future development of the ICR Grid, the results for the 3-X-5 tasks in Sessions 6 and 7 were also poorer than in the other sessions, suggesting that on a broader level team composition may have been a factor. The teams were randomly assigned and it could simply be the case that those sessions had weaker students assigned to them. The personalities in the team may not have gelled, leading to poor dynamics. Also, the language skills of some of the students in those teams were poorer than others, inhibiting the quality of communication between team members. These sessions were two of the three which indicated they enjoyed the 6-3-5 Method more than the ICR Grid - the other teams showed a preference for using the ICR Grid.

Considering the overall profile of the concepts produced (averaged for all eight sessions) is illustrated in Figure 9. The quantity of concepts produced by the 3-X-5 
Method is clearly greater, despite consistently falling short of the optimum three concepts for every five minute round of the task. During the 3-X-5 task, at times participants seemed to be drawing ideas for the sake of it, with similar themes noticeably repeated towards the end. If the purpose of a concept design session is to produce a large number of ideas, then it is important that there is sufficient focus and scope to sustain the team's effort throughout. If the combination of personalities is not right and the quality of concepts begins to wane, there is little scope in the $3-\mathrm{X}-5$ Method for re-invigorating proceedings. The ICR Grid's emphasis on providing stimuli through the task helped to give fresh impetus at times but the downside of this was that it did not have scope for the rapid iteration of the 3-X-5 Method. This was accepted as a consequence of allocating a portion of the task time to search activities in the hope that the range of activities would ensure that the output would overall be more robust.
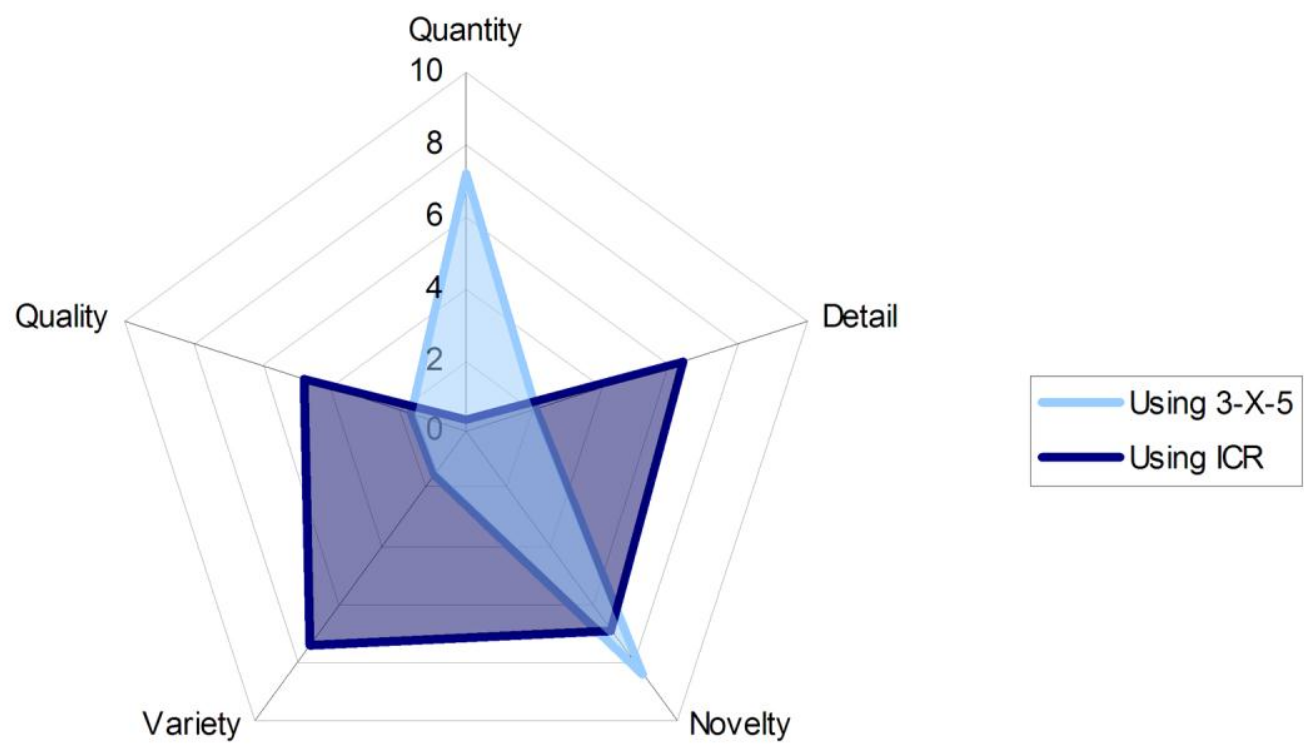

Figure 9: Summary of results

Participants in both tasks were asked to sketch at a speed that felt comfortable to them, so even if they did not produce the projected three concepts per five minutes for the 3-X-5 task, they should have completed sketches with a comparable level of detail to those in the ICR Grid. In actuality, the concepts produced in the ICR Grid generally showed better attention to detail. An attributable factor is participants 
having the opportunity to examine and utilise reference mechanisms, details and forms from existing competitor and pertinent designs.

The 3-X-5 concepts scored marginally higher in terms of novelty, and as a measure of how different each concept was from another this reflects its more open-ended approach compared to the ICR Grid. The previous ideas to which participants had access as the task progressed could be freely used or discarded as new concepts were produced. In the ICR Grid, participants were at times required to build directly on a concept if it had been identified as promising, thereby limiting the scope for a high novelty score with the resulting concept sketch. As the 3-X-5 tasks progressed, it was obvious that the concepts created were heavily referred to during the sessions and as a result the novelty score for $3-\mathrm{X}-5$ is only marginally higher than that for the ICR Grid. This shows a tendency for participants to be influenced by the thinking of others.

The score for variety is considerably better for the ICR Grid. As a measurement applied to the group of concepts, this indicates that despite a smaller pool of concepts, a proportionately higher number of different principles were used. In the ICR Grid, a good range of different principles of operation identified and then variations of these subsequently created. Again, this can be attributed to participants having the opportunity to explore relevant information and suggest appropriate solutions. The 3-X-5 tasks tended to be more haphazard in that new configurations would occasionally be introduced, but then small variations would be applied continually without necessarily taking the concept anywhere new.

The overall rating for quality was a combination of an evaluation against requirements and detail of the concepts. The evaluative scoring took into account both originality and feasibility, but the quality score can best be viewed as an overview of concept viability. It can be argued that as a measure of quality this does not sufficiently reward the level of creative thinking in the concepts, but novelty and variety scores have been used to provide more insight into these specific aspects. Again, the ICR Grid scored noticeably higher. This can be linked to the higher detail documented for the concepts of the ICR Grid. Additionally, the ICR Grid was more 
explicit in asking participants to address the requirements in the brief, so the concepts produced were more likely to satisfy these.

Overall, it was found that the ICR Grid produced better rounded concepts than the 3-X-5 Method, scoring more highly across the criteria. However, the 3-X-5 Method did produce more concepts with a slightly better novelty value. This suggests that the method lends itself better to an earlier phase in the design process where the team wish to simply explore a range of high level ideas unconstrained by design requirements and without emphasis on trying to develop robust concepts. The best of the ideas produced in such a session could easily be compiled to form one of the inputs to the ICR Grid.

\subsection{Information retrieval and utilisation}

As a key differentiating factor between the 6-3-5 Method and ICR Grid, the information retrieval and utilisation during the sessions was reviewed. The laptops used were a number of years old and limited in computing power, but in the end a number of useful information resources were constructed in OneNote despite the restricted speed of response. The average number of sources found was 10 for the $30-$ minute sessions, with all information sources coming through Google searches. It is anticipated that given a greater timeframe, physical sources such as textbooks, models and sample material could be captured through the use of digital cameras or scanning and inputted to the grid in a similar manner. A screenshot of the grid from Session 3 is shown in Figure 10. 


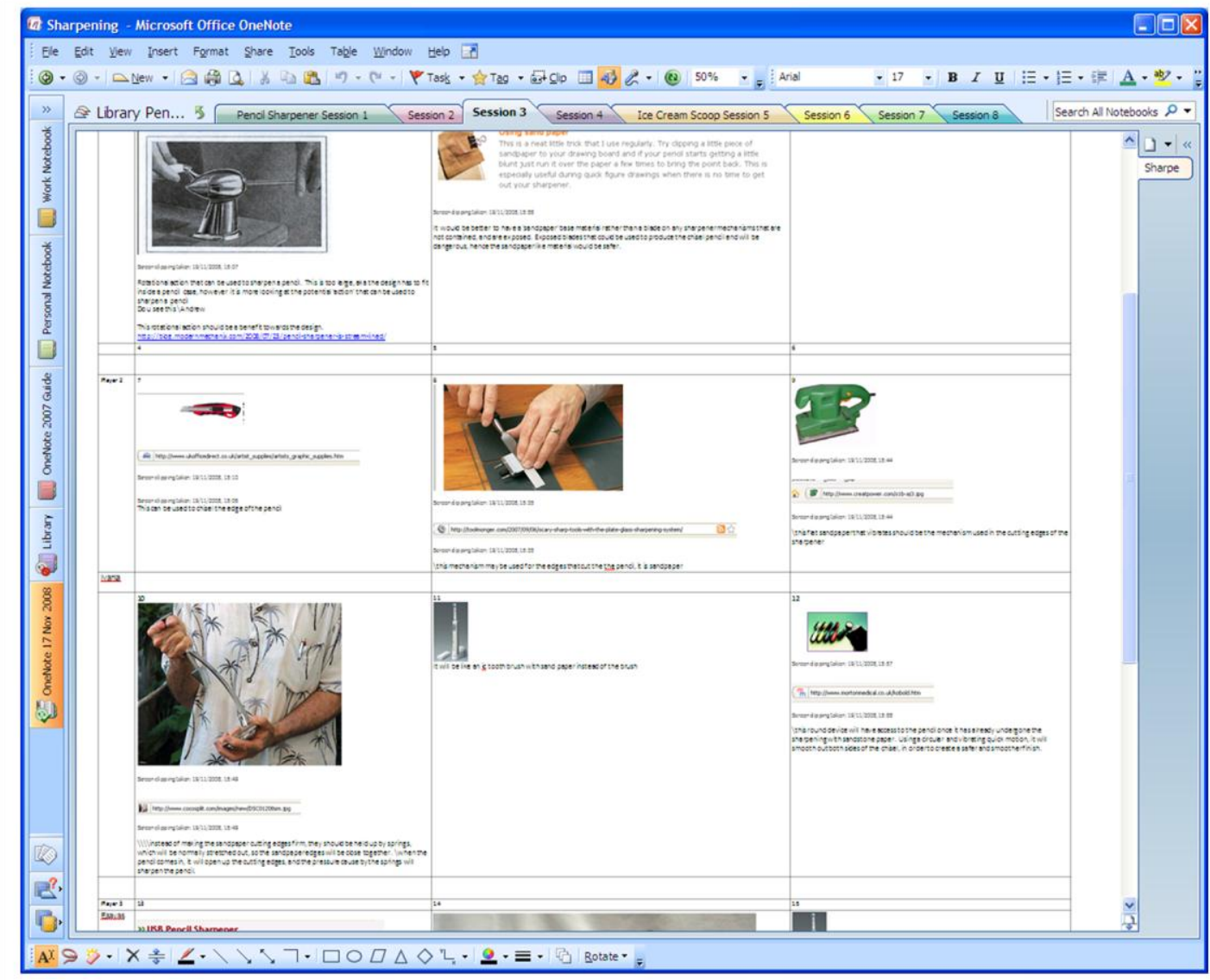

Figure 10: Screenshot of OneNote library for Session 3

From Vincenti's (1990) taxonomy of design knowledge, the majority of items retrieved (70 of 84$)$ were identified as fundamental design concepts, consisting of representations of existing principles, configurations or structures. These were additionally delineated as internal or external to the domain of application (Howard 2008), with the majority (45 of 70) proving to be to images of products, either direct competitors or devices using mechanisms which may be applicable. The results also indicated a direct relationship between the number of information items found during a session and the average quality of output. While this may have been because the information had a positive effect on the work produced, it could also have been due to high performing groups.

Despite a concern prior to the sessions taking place that there may be a resistance to the requirement in the ICR Grid to undertake searching tasks at the expense of concept sketching, it was found that there was a preference for this among the participants. On questioning, this was revealed to be associated with confidence: 
participants felt that having the facility to consult information as necessary meant that the concepts subsequently generated would be better substantiated. Although the information sources did provide stimulus for concepts, there was a concern that difficulty in finding good quality and diverse sources could inhibit the associated conceptual work. In terms of Information Literacy (IL), finding competitor products (internal stimuli) can be rated the easiest type of information to source: simply using the product name is enough to return results on related products. Finding different, but potentially relevant, products or technologies (external stimuli) requires the participant to think about possible features or major specifications relating to the design, with search results typically providing more tangential information. More sophisticated behaviour is shown when participants identify the underlying characteristics and principles that could be adopted, and interpret how these could be applied. These aspects of information use have been identified for further investigation in future implementations of the ICR Grid.

\section{Conclusions}

This work has described an augmented version of the 6-3-5 Method, the ICR Grid, which incorporates the systematic use of information. In a comparative study, the ICR Grid was well-received by participants and performed better in terms of producing concepts of superior quality, variety and detail. The integrated 'research, create, evaluate' approach was found to be effective in bringing information to bear on concept design and positively affected the quality of concept work. Its approach to generating and linking information resources as part of the conceptual design work suggests a new model to improve the effectiveness of digital libraries and information resources in the design process as well as compressing previously discrete stages in the concept design phase. Although grounded in the area of product development, this research is applicable to any organisation undertaking idea generation and problem solving. A set of industrial studies have subsequently been conducted examining the performance of the method in three different company contexts (Wodehouse and Ion 2010b), with the results indicating that the method was flexible enough to adapt to different situations. As well as providing insights on the transference of information and research to conceptual design activity, this work 
holds additional interest for studies on the composition and use of digital libraries in design. Areas for future development for the ICR Grid include enhancing the digital environment and investigating in more detail patterns of information use.

\section{References}

Asimow, M., 1962. Introduction to design Englewood Cliffs, NJ: Prentice-Hall.

Benami, O. \& Jin, Y., Year. Creative stimulation in conceptual designed.^eds. ASME 2002 Design Engineering Technical Confereces and Computer and Information in Engineering Conference, Montreal, Canada, DETC2002/DTM-34023.

Casakin, H.P., 2007. Factors of metaphors in design problem-solving: Implications for design creativity. International Journal of Design, 1 (2), 21-33.

Chuang, Y. \& Chen, L.L., 2008. How to rate 100 visual stimuli efficiently. International Journal of Design, 2 (1), 31-43.

Cross, N., 1994. Engineering design methods, strategies for product design Chichester, UK: John Wiley \& Sons.

Demian, P. \& Fruchter, R., 2009. Effective visualisation of design versions: Visual storytelling for design reuse. Research in Engineering Design, 19, 193-204.

Eris, O., 2004. Effective inquiry for innovative engineering design Dordrecht, the Netherlands: Kluwer Academic Publishers.

French, M., 1985. Conceptual design for engineers London, UK: The Design Council/ Springer-Verlag.

Gero, J.S. \& Kannengiesser, U., 2004. The situated function-behaviour-structure framework. Design Studies, 25 (373-391).

Goldschmidt, G., 1991. The dialectics of sketching. Creativity Research Journal, 4 (2), 123 143.

Hellfritz, H., 1978. Innovation via galeriemethode (innovation via the art gallery method) Koenistein im Taunus, Germany: Eigenverlag.

Howard, T., 2008. Information management for creative stimuli in engineering design. University of Bath.

Kelley, T., 2006. The ten faces of innovation: Strategies for heightening creativity London, UK: Profile Books.

Kulkarni, S., Summers, J.D., Vargas-Hernandez, N. \& Shah, J.J., 2001. Collaborative sketching (c-sketch) - an idea generation technique for engineering design. The Journal of Creative Behavior, 35 (3), 168-198.

Osborn, A., 1953. Applied imagination: Principles and procedures of creative problem solving New York, NY: Charles Scribner's Sons.

Pahl, G. \& Beitz, W., 1995. Engineering design, a systematic approach Bath, UK: Springer.

Pugh, S., 1991. Total design Reading, UK: Addison-Wesley.

Rodgers, P.A., Green, G. \& Mcgown, A., 2000. Using concept sketches to track design progress. Design Studies, 21, 451-464.

Rohrbach, B., 1969. Kreativ nach regeln. Absatzwirtschaft, 12, 73-75.

Shah, J.J., Kulkarni, S.V. \& Vargas-Hernandez, N., 2000. Evaluation of idea generation methods for conceptual design: Effectiveness metrics and design of experiments. Journal of Mechanical Design, 122 (4), 377-385.

Shah, J.J. \& Vargas-Hernandez, N., 2003. Metrics for measuring ideation effectiveness. Design Studies, 24, 111-134.

Sim, S.K. \& Duffy, A.H.B., 2003. Towards an ontology of generic engineering design activities. Research in Engineering Design, 14, 200-223. 
Smith, S.M., Kohn, N.W. \& Shah, J., Year. What you see is what you get: Effects of provocative stimuli in creative inventioned.^eds. NSF International Workshop on Studying Design Creativity, Provence, France,

http://mason.gmu.edu/ jgero/conferences/sdc08/papers/Smith.pdf (Accessed 12th June 2009).

Sutton, R.I. \& Hargadon, A., 1996. Brainstorming groups in context: Effectiveness in a product design firm. Administrative Science Quarterly, 41 (4), 685-718.

Ulrich, K.T. \& Eppinger, S.D., 1995. Product design and development, 3rd (International) Edition ed. New York, NY: McGraw-Hill.

Vincenti, W.G., 1990. What engineers know and how they know it: Analytical studies from aeronautical history Baltimore, MA: John Hopkins.

Wodehouse, A.J. \& Ion, W.J., 2010a. Digital information support for concept design. CoDesign, 6 (1), 3-23.

Wodehouse, A.J. \& Ion, W.J., 2010b. Industrial application of a new conceptual design method: Enhancing information use with the icr grid. Journal of Design Research (under review). 